\title{
Das Pantokymographion.
}

\author{
Von \\ Th. W. Engelmann.
}

Hierzu Tafel I und II.

Die bisher beschriebenen und in den physiologisehen Laboratorien gebräuchlichen Registrirapparate haben das Gemeinsame, dass jeder nur für eine verhältnissmässig beschränkte Zahl von Zweeken zu brauchen ist. Sie erlauben entweder nur Erscheinungen von sehr kurzer Dauer (Myographion mit Ubrwerk von Helmholtz, Pendelmyographion von Fick, Helm holtz, Federmyographion von E. du Bo is - Re ymond u. A.) oder verhältnissmässig langsam verlaufende Bewegungen während kürzerer oder längerer Zeit zu registriren 'Kymographion von Ludwig mit rotirendem Cylinder, mit Papier ohne Ende und andere Modificationen); andere gestatten nur die Verwendung einiger weniger bestimmter Schnelligkeiten der Schreibfläche (Registrirapparat von Marey mit Foucault's Regulator). Nur wenige sind mit automatisch arbeitenden Vorrichtungen für die so oft erforderliche Anwendung electrischer Ströme versehen, und auch diese Vorrichtungen sind nur für ganz bestimmte Fälle berechnet.

Es schien möglich, ohne der Genauigkeit der Leistungen und der Bequemlichkeit der Handhabung Abbruch zu thun, die den verschiedenen Registrirapparaten eigenen Vortheile zu vereinigen und zugleich die grösste Vielseitigkeit der Vorrichtungen zu automatischer Schliessung und Oeffnung von electrischen Leitungen zu erreichen, wenn an dem gewöhnlichen Kymographion mit grossem rotirenden Schreibeylinder einmal ein Federmechanismus zur Erzeugung einmaliger Umdrehungen von grosser Geschwindigkeit und zweitens das rhythmische Polyrheotom ${ }^{1}$ ) als integrirende

1) Das rhythmische Polyrheotom. Dies Archiv Bd. 52. 1893. p. 603. Taf. IV. - Das Princip der gemeinschaftlichen Strecke. Ebenda p. 592. 
Bestandtheile angebracht würden. Die Verfolgung dieses Plans führte zur Construction des Pantok y mographion, dessen Einrichtung und Leistungen hier kurz beschrieben werden mögen, da lang fortgesetzter Gebrauch gezeigt hat, dass sie den gestellten sehr hohen Anforderungen durchaus genügen und ich häufig bei der Mittheilung von Versuchen in der Lage sein werde, die Bekanntschaft mit dem Instrument vorauszusetzen.

Eine ausführlichere, von zahlreichen Abbildungen begleitete Beschreibung des Apparates und seiner einzelnen Theile, sowie eine eingehende Experimentalkritik desselben hat Herr W. A.

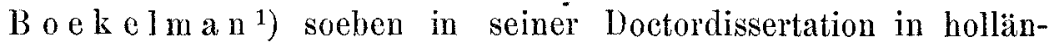
discher Sprache gegeben. Ausserdem ist die Brauchbarkeit des Instruments speziell für feinere Zeitmessungen dargethan in einer Untersuchung über die Leitungsgeschwindigkeit des Reizes in den marklosen Nervenfibrillen der Cornea und in einer grossen Reihe von Messungen der Leitungsgesehwindigkeit in motorischen Nerven, deren Resultate theilweise bereits im Holländischen veröffentlicht wurden ${ }^{2}$ ).

\section{Allgemeine Einrichtung des Apparats.}

Das Pantokymographion hat im Wesentlichen dic Form und die Dimensionen des im hiesigen Laboratorium seit Jahren gebräuchlichen, auch in vielen anderen Instituten eingeführten allgemeinen Registrirapparats, welcher in der Hauptsache nach dem Princip des älteren Ludwig'schen Kymographions mit grossem rotirenden Cylinder gebaut ist. Taf. I giebt eine Vorstellung.

Ein fester 'Tisch von Mahagoniholz, $0,70 \mathrm{~m}$ boch, trägt auf einer $55 \mathrm{~mm}$ langen, $42 \mathrm{cmm}$ breiten Grundplatte $A$ den Registrirund Reizapparat. Der Tisch ruht auf vier soliden Füssen, die in etwa $35 \mathrm{~mm}$ Höhe über dem Boden durch eine kräftige Holzplatte fest verbunden sind. Schrauben in den Fïssen gestatten die Platte $A$ fest und horizontal einzustellen.

1) W. A. Boekelman, Het pantokymographion en eenige daarmede verrichte physiologische proeven. Proefschrift (Utrecht 1894). 58 Seiten, 7 Tafeln und mehrere Holzschnitte. - Das Instrument wird rom Mechaniker des physiologischen Instituts D. B. Ka gen a a r, zum Preise von $500 \mathrm{fl}$. holl. geliefert.

2) Boekelman, a. a. O. und Th. W. Engelmann im Proc. verb. d. k. Akad. v. wetensch. te Amsterdam. Afd. Natuurk. Zitting van 24. Nov. 1894. 
Auf der Mitte der Grundplatte $A$ befindet sich in einem seitlich durch Glasplatten, unten und oben durch starke, an den Kanten durch Messingsäulen verbundene, Messingplatten abgeschlossenen Raum das Uhrwerk, welches durch die Gewichte $L$ in Bewegung zu bringen ist. Zum Aufwinden dient die unter der Tischplatte angebrachte Kurbel $G$.

Das Uhrwerk wirkt auf eine massive stählerne Hauptaxe, welche $5 \mathrm{~cm}$ ïber das Gehäuse des Uhrwerks hervorragt und oben in eine konische Spitze ausläuft, auf welche das konisch ausgebohrte untere Ende der den Schreibcylinder $D$ tragenden $A x e E$ aufgesetzt wird. In das obere gleichfalls konisch ausgebohrte Ende der letzteren Axe passt die Spitze einer Stahlschraube, welche in einer Mutter am freien Ende des horizontalen gusseisernen Armes $C$ der schweren, auf der Tischplatte $A$ rechts sich erhebenden, gleichfalls gusseisernen säule $B$ läuft.

Auf die Hauptaxe des Uhrwerks kann . die Scheibe $N$ des Polyrheotoms aufgeschoben werden. Sie trägt auf ihrer oberen Fläche den Mitnehmer, eine vertikale, oben "offene, durch eine Schraube anzuziehende Klemme, in welche beim Aufsetzen der Cylinderaxe $E$ ein am unteren Ende der letzteren befestigter horizontaler stählerner Arm eingelegt wird. Bei Drehung der Axe $E$ wird dann die Scheibe $N$, bei Drehung von Scheibe $N$ die Axe $E$ mitgenommen.

Vor dem Gehäuse des Uhrwerks, an der Seite des Experimentators, ist anf der Grundplatte $A$ die früher ${ }^{1}$ ) ausführlich beschriebene and abgebildete Doppelcontactvor r c htu $\mathrm{g}$ des Polyrheotoms, vertical und horizontal verstellbar angebracht, in solcher Weise, dass dieselbe innerhalb der nämlichen Grenzen wie beim urspriinglichen Polyrheotom functioniren kann.

An dem metallenen Gehäuse des Uhrwerks sitzen links zwei Doppelklemmschrauben zur Aufnahme von Drähten electrischer Leitungen. Die Metallmassen des Gehäuses, des Uhrwerks und der Rheotomscheibe bilden die ,gemeinschaftliche Strecke" des Polyrheotoms, deren Widerstand schon unter gewöhnlichen Verhältnissen nicht merklich in Betracht kommt und, wo dies etwa der Fall sein sollte, stets durch Einschaltung von Widerständen an anderen Stellen der Leitungen practisch zu Null gemacht werden kann.

1) Das rhythmische Polyrheotom a. a. 0. p. 605. Taf. IV. 
Auf der Grundplatte $A$ erheben sich noch zwei starke bohle Messingsäulen $F$, in welche ein grösseres oder zwei kleinere Bretter $Q$ unbeweglich, aber horizontal und vertical verstellbar festgeschraubt werden können. Sie dienen zum Aufstellen der Schreibapparate und sonstiger Hilfsvorrichtungen. In der Figur ist nur eins der kleineren Bretter abgebildet.

Der Registrircylinder von $60 \mathrm{~cm}$ Umfang und $30 \mathrm{~cm}$ Höhe - in üblicher Weise mit einem Bogen Glanzpapier fest bespannt, der über einer breiten qualmenden Petroleumflamme berusst wird kann entweder durch einen Federmechanismus oder durch das Uhrwerk, iibrigens auch mit der freien Hand in Umdrehung versetzt werden. An Stelle des Uhrwerks würde da, wo eine hin. reichend kräftige und gleichmässige andere Triebkraft (Gasmaschine, Wasser- oder electrischer Motor) zur Verfügung steht, nach Anbringung geringfügiger Aenderungen auch diese benutzt werden können. Immerhin gewähren Uhrwerk und Federmechanismus den Vortheil, dass der Apparat an jedem beliebigen Orte aufgestellt und zu allen überhaupt zulässigen Zwecken ohne Weiteres benutzt werden kann.

\section{Der Federmechanismus und seine Wirkung.}

Derselbe dient zur Erzeugung einmaliger Umdrehungen von so grosser Geschwindigkeit, dass sehr schnell verlaufende, kurz danernde Vorgänge registrirt und damit äusserst kleine Zeittheilchen - bis unter $1 / 20000$ Sekunde herab -- auf graphischem Wege gemessen werden können. Er ist folgendermaassen eingerichtet ${ }^{1}$ ).

Am Ende des horizontalen massiven Armes $C$ des gusseisernen Stativs ist eine Messingscheibe fest aufgeschraubt, auf deren Mitte sich ein kurzer starker hohler Messingeylinder erhebt, welcher die Axe für die Federtrommel $O$ bildet. Durch ihn und durch die Messingscheibe geht zugleich die zur oberen Fixirung der Axe des Schreibcylinders bestimmte Schraube. Die cylindrische Federtrommel, gleichfalls von Messing, ist unten durch eine Messingplatte, oben durch einen abschraubbaren Deckel geschlossen und enthält im Innern aufgerollt eine $2 \mathrm{~m}$ lange, $3 \mathrm{~cm}$ breite, $0,4 \mathrm{~mm}$ dicke Feder von bestem Stahl. Das eine Ende dieser Feder ist

1) Ausführliche Beschreibung und Abbildung bei Boekelman p. 4-8, 17-22, Taf. II u. Taf. VI. Fig. 1-4. 
an der Innenseite der äusseren Trommelwand unbeweglich angeschraubt, das andere wird bei Drehung der Trommel zum Zwecke des Spannens der Feder von einem Zahn erfasst und fixirt, der an der unbeweglichen cylindrischen Axe festsitzt. Unten hat dieTrommel eine kräftige Flantsche, in welcher sich in gleichen Winkelabständen 12 cylindrische, numerirte Löcher befinden. Diese dienen zur Anfnahme und Befestigung eines, bezüglich zweier, starker stählerner vertikaler Stifte, des „Treibstifts" und des „Fangstifts".

Der Treibstift wird bei gespannter Feder gegen einen starken horizontalen Stahlstift den D r u ckst ift, angepresst, welcher am oberen Ende der den Schreibcylinder tragenden Axe aufgeschraubt werden kann. In der Anfangs- und Ruhestellung des Cylinders wird dieser Stift und damit die Axe des Schreibeylinders durch einen Zahn festgehalten, welcher sich an einer am Arm $O$ befestigten um eine horizontale Axe drehbaren starken Hebelvorrichtung befindet. Wird durch einen kurzen Druck von unten auf den Arm $P$ des Hebels dieser Sperrzahn weggeschoben, so geräth der Cylinder sofort in Bewegung. Eine Spiralfeder an der Säule $B$ des Stativs bringt beim Loslassen von $P$ den Hebel und damit den Sperrzahn sofort in die Anfangstellung zurïck. Kurz vor Vollendung des Umlaufs wird durch eine regulirbare Hemmung - zwei starke verstellbare Federn, zwisehen welchen der Druckstift der Cylinderaxe mit Reibung hingleitet die Bewegung des Cylinders so verzögert, dass er mit etwa der Geschwindigkeit Null die Anfangstellung erreicht, wo er dann wieder gegen den Sperrzahn anliegt.

Die Dauer einer Umdrehung und die während derselben erreichte grösste Geschwindigkeit hängt in jedem Falle von der Anfangsspannung der Feder $a b$ und diese von der Stelle des Trommelumfangs, an welcher der Treibstift in die Flantsche eingesetzt war. Da man diese Stellung 12 mal ändern und bis zu mehr als $2^{1} / 2$ Umdrehungen der Federtrommel ausnutzen kann, verftugt man über mehr als 30 verschiedene Anfangsspannungen. Die vier schwächsten geniigen nicht $z \mathfrak{u}$ einer vollständigen Umdrehung des Cylinders. Ein besonderer Druckstift gestattet hier die Feder auf einem Umgang zweimal zu verwenden.

Wie aus der Beschreibung erhellt, nimmt die Geschwindigkeit des Cylinders zu, so lange die Federtrommel mittelst des Treibstifts auf den Druckstift der Cylinderaxe wirkt. Die Dauer 
dieser Einwirkung kann nun mit Hilfe eines zweiten Stifts, des Fangstifts, verkïrzt werden, der zu dem Ende in der nämlichen Weise wie der Treibstift in eines der Löcher in der Flantsche der Federtrommel eingesetzt und nach einer gewissen Winkeldrehung der Axe zwischen zwei am Arm $C$ des Stativs befestigten starken Federn aufgefangen wird. Je weiter vor dem Treibstift der Fangstift eingesetzt wurde, desto kleiner ist die Drehung, nach welcher dies Auffangen erfolgt, um so eher hört also die Beschleunigung des Cylinders auf. Letzterer dreht sich dann weiter mit einer Geschwindigkeit, die anfangss sehr langsam, dann, sobald der Druekstift zwischen die Federn der Hemmung geräth, schnell abnimmt.

Je nach Stellung des Treibstifts (Anfangssspannung) und des Fangstifts (Daner der Federwirkung) variirt die Dauer einer Rotation zwischen etwa 0,5 und 2 Secunden, und die maximale, während derselben exreichte Geschwindigkeit der Schreibfläche zwischen etwa $275 \mathrm{~mm}$ und über $2000 \mathrm{~mm}$. Lässt man den Fangstift ganz weg, so kann die Geschwindigkeit noch höher gesteigert werden; dasselbe wiirde sich auch durch Verstärkung der Feder und Verminderung des Gewichts der rotirenden Masse (Axe, Cylinder, Rheotomscheibe) erreichen lassen.

Bei meinem Apparat werden beispielsweise bei Benutzung einer Axe von $0,65 \mathrm{k}$, einem Cylinder von $1,7 \mathrm{k}$ und einer Rheotomscheibe von $0,8 \mathrm{k}$ Gerwicht, bei Weglassung ides Fangstifts die folgenden maximalen Geschwindigkeiten erhalten:

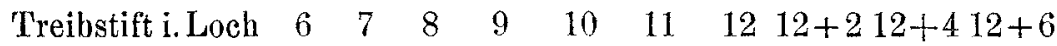
Max. Geschwindigkeit in $\mathrm{mm}$ $72075097511001210126013801550 \quad 1650 \quad 1760$

Die genaue Bestimmung der Geschwindigkeit in jedem kleinsten Moment während der Umdrehung erfolgt durch gleichzeitiges Registriren einer Stimmgabel von hinreichend boher Schwingungszahl, am besten mittels Lufttransport oder electrischen Signals. Bei derselben Anfangsspannung der Feder, und bei Benutzung der gleichen Axe, des nämlichen Cylinders a. s. w. genügt im Allgemeinen eine einmalige Bestimmung. Doch können folgende Umstände einen Einfluss ausüben: die Fixation der Cylinderaxe auf der Rheotomscheibe durch den Mitnehmer, die Reibung der Kupfercontacte des Polyrheotoms, die Reibung der auf dem Cylinder schreibenden Federspitzen.

L. Pfinger, Archiv f. Physiologie. Bd. 60. 
In Bezug auf ersteren Punkt ist im Interesse einer gleichmässigen und schnelleren Bewegung zu empfehlen, den horizontalen Arm am unteren Ende der Cylinderaxe nicht unbeweglich zwischen den Armen des Mitnehmers festzuschrauben, sondern ihm einen, wenn auch nur ganz kleinen Spielraum (etwa $0,1 \mathrm{~mm}$ ) zu lassen. Näheres hiertiber gibt Boekelm an auf p. 20 u. Tab. II.

Die Reibung der Contacte des Polyrheotoms macht sich im Allgemeinen nur bemerklich bei gleichzeitiger Verwendung einer grösseren Zahl von Federcontacten und bei geringer Geschwindig. keit des Cylinders. Wenn man aber durch genaue Einstellung der Contactbänke nnd der Contactfedern sorgt, dass die Reibung nicht viel grösser wird als zur Herstellung sicheren und gleichmässigen Contacts überhaupt erforderlich ist, so lässt sich dieser Einfluss völlig unbemerkbar machen.

Die Reibung der schreibenden Spitzen auf dem berussten Glanzpapier hat noch weniger Einfluss. Man findet auch hiertiber bei Boekelman (p. 21 und 22) genane Angaben.

Es versteht sich von selbst, dass bei Benutzung der Federmechanik die Rheotomseheibe nicht gegen die Hauptaxe des Uhrwerks angeschraubt, sondern auf diese nur aufgeschoben wird, da sie sich ja um dieselbe drehen muss.

\section{Die Bewegung durch das Uhrwerk.}

Soll das Uhrwerk den Cylinder in Bewegung bringen, so fixirt man die Rheotomscheibe auf der Hauptaxe des Uhrwerks durch leises Anziehen der Schraube und setzt die den Cylinder tragende Axe in ihre Lager. Der Druckstift wird weggelassen, der untere horizontale Arm der Axe mit einigem Spielraum in den Mitnehmer eingesetzt. Fiir den Fall die Rheotomscheibe nicht benutzt werden soll, kann sie entfernt und ein eigener kleiner Mitnehmer an ihrer Stelle auf die Hauptaxe des Uhrwerks befestigt werden.

Die Auslösung der Bewegung geschieht durch Wegdrücken einer auf der Axe der Windfligel schleifenden Feder. Durch den Einfluss der Windflügel wird die anfangs sich beschleunigende Bewegung bald merklich constant. Die Gleichförmigkeit der Bewegung ist im Allgemeinen um so grösser, je schwerer die treibenden Gewichte. Von Wichtigkeit in dieser Hinsicht ist aber namentlich wiederum, dass die Cylinderaxe im Mitnehmer einigen Spiel- 
raum habe und ausserdem, dass die Rheotomscheibe, falls sie zur Verwendung kommt, nicht fester als für sicheres Mitnehmen durchaus nöthig, auf der Hauptaxe des Uhrwerks festgeschraubt werde. Wird hierfïr gut gesorgt, so entspricht die Constanz der Bewegung bei den verchiedensten absoluten Geschwindigkeiten allen billigen Anforderungen.

Die Geschwindigkeit der Bewegung mittels des Uhrwerks kann auf vier verschiedene Weisen variirt werden: durch Vermehrung oder Verminderung der Gewichte $L$ (Taf. I), zweitens dadurch, dass man die die Gewichte tragende Saite entweder über nur eine Rolle (3) oder - im Flaschenzug - iber 2 ( $I$ und $H$ ), oder $3(I, H$ und $K$ ) Rollen laufen lässt; drittens mittels eines Räderwechsels (durch Heben oder Senken eines hinter dem Gehäuse des Uhrwerks auf der Tischplatte $A$ hervorragenden Stifts); endlich durch Verstellung oder Wechsel der Windfligel, von denen ein kleines Paar iiber, ein grosses $(M)$ unter der Tischplatte $A$ angebracht ist. Durch Verrïcken eines auf dem Deckel des Radgehäuses befindlichen Messingschiebers kann nach Belieben das kleine oder grosse Paar mit dem Uhrwerk verbunden werden ${ }^{1}$ ).

Es lassen sich auf diesem Wege alle möglichen, zwischen etwa $0,5 \mathrm{~mm}$ und $250 \mathrm{~mm}$ in der Secunde liegenden constanten Geschwindigkeiten herstellen, und zwar eine jede auf mehrfache Weise, durch verschiedene Anwendung und Combination jener vier Factoren ${ }^{2}$ ). Die Dauer einer einzelnen Cylinderumdrehung lässt sich also zwischen etwa $2^{1 / 2}$ Secunden und 20 Minuten variiren, was für die meisten Versuchszwecke geniigt.

Es ist aber auch möglich, während noch viel längerer Zeit zu registriren, ohne dass der Experimentator den Cylinder auf der Axe zu verschieben oder überhaupt einzugreifen, ja ohne dass er auch nur gegenwärtig zu sein braucht. Hierzu dienen die folgenden automatisch wirkenden Einrichtungen:

a) Die Einrichtung für Spir al bew egung des Cylinders. Sie bewirkt, dass der Cylinder, während er durch das Uhrwerk in Umdrehung gesetzt wird, zugleich längs der Axe nach abwärts

1) Die genauere Beschreibung s. bei Bo ekelman p. 8 u. flg. Taf. VI, Fig. 5.

2) Nähere Zahlenangaben mit Rücksicht auch auf die Constanz bei Boekelman, p. 23 u. 24. 
gleitet, jeder Punkt seiner Oberfläche also nicht eine in sich geschlossene Kreislinie, sondern eine Spirale beschreibt. Es kommt hierbei eine hohle Axe und ein eigener Schreibcylinder zur Verwendung, von beiläufig denselben Dimensionen wie der gewöhnliche. Ehe dieselben eingesetzt werden, schraubt man die die Federtrommel tragende Messingscheibe los, entfernt diese und die Federtrommel und befestigt an ihrer Stelle (mit der nämlichen Scbraube) eine Rolle auf dem Arm $C$. Ueber diese Rolle und über eine nahe dem anderen Ende von $C$ befestigte zweite Rolle (auf Taf. I sichtbar) läuft eine Schnur, die durch ein Loch im Arm $C$ hindurch nach abwärts, längs der Säule $B$, und nahe deren unterem Ende über eine dritte Rolle nach einer Spule gefübrt wird, an der ihr eines Ende eingehakt und um welche sie durch Drehung der Axe, auf welcher die Spule sitzt, aufgewunden wird. An der Axe der Spule sitzt ein Zahnrad, welehes mittelst eines Schiebers so eingestellt werden kann, dass es von einem Zahnrad des Uhrwerks gefasst und bei dessen Umdrehung so mitgenommen wird, dass die Schnur sich dabei von der Spule abwickeln muss. Am anderen Ende der Schnur hängt nun der Cylinder mittels eines an der Schnur befestigten stählernen Stäbchens, welches durch eine hoble, als oberes Lager für die Cylinderaxe dienende Schraube in der Mutter von Arm $C$ hindurch in die hohle Axe des Cylinders geschoben und hier in einem vertical durchbohrten Messingprisma festgeschraubt wird, welches auf der oberen Fläche des Schreibcylinders verstellbar befestigt, durch einen seitlich in und längs dessen Axe verlaufenden Schlitz in die Höhlung der Axe eingeschoben werden kann. Der Cylinder, der nicht auf der Axe festgeschranbt werden darf, hängt auf diese Weise frei an dem im Innern der Axe schwebenden Stift und gleitet nun während der Umdrehung des Cylinders in Folge der Abwicklung der Schnur von der Spule allmählich längs der Axe nach abwärts. Je nack dem Durchmesser der Spule erfolgt das Herabsinken schneller oder langsamer und sind dementsprechend die Neigung und der verticale Abstand der auf einem Cylinder übereinander gezeichneten Spiraltouren versehieden. Es werden drei Spulen beigegeben, die ausgewechselt werden können und für welche die Neigungswinkel der Spirale, resp. etwa $1^{0}, 1,5^{0}$ und $2^{0}$, die Zahl der auf einen Bogen entfallenden Spiraltouren etwa 30, 18 und 12 beträgt. Bei der geringsten Rotationsgeschwindigkeit und Benutzung der kleinsten 
Spule kann demnach $30 \times 20$ Minuten, d. i. 10 Stunden lang fortlaufend auf dem nämlichen Bogen registrirt werden, und zwar ohne dass die Gewichte aufgezogen zu werden brauchen.

b) Einrichtung für periodische Arretirung des Cylinders. Eine noch beliebig viel längere Versuchsdauer, ohne Wechsel des Cylinders, wird ermöglicht dureh einen am Gehäuse des Uhrwerks angebrachten kleinen Electromagneten, welcher bei Schliessung seines Stromes durch Herabziehen eines horizontalen Hebels mit verstellbarer Spitze die Bewegung der kleinen Windflügel und damit des Cylinders hemmt, bei Oeffnung wieder freilässt. Durch einen Metronom oder ein geeignetes Uhrwerk können Dauer und Intervalle der Stromschliessungen und damit Dauer und Intervalle der Bewegungen des Cylinders, wie man leicht einsieht, beliebig variirt werden. Der Mangel einer wirklich continuirlichen Bewegung dürfte hier nicht leicht empfunden werden, da es bei tage- oder wochenlang fortgesetzten graphischen Versuchen und Beobachtungen ja kaum jemals auf absolut continuirliches Registriren, sondern nur auf ein periodisches Verzeichnen des Standes, bezüglich der Bewegungen der Schreibspitzen, eventuell auf Verzeichnen der während gewisser Zeitränme vorkommenden Maxima und Minima ankommt.

\section{Verbindung des Polyrheotoms mit dem Kymographion.}

Dureh die Verbindung des rhythmischen Polyrheotoms mit dem Kymographion wird dieses zugleich eine Reizvorrichtung von vielseitigster Verwendbarkeit. Fs gewährt demgemäss zunächst alle die Vortheile, welche das Polyrheotom an und für sich bietet und wofür früher Beispiele und Belege mitgetheilt wurden. Eine Erweiterung seiner Leistungen hat letzteres ausserdem erhalten dureh Zugabe eines Ebonitbänkchens mit horizontal beweglichem Stromschlissel, der durch einen in die Rheotomscheibe einzusetzender Zapfen im Vorbeigehen geöffnet werden kann. Hierdurch wird es möglich, ohne Vithilfe einer Abblendungsvorrichtung nur Schliessungen oder nur Oeffnungen galvanischer Ströme zu verwenden und ihre Momente genall zul registriren.

Ein nicht genug zu sehätzender Vorzug liegt aber weiter darin, dass die Momente der Schliessung und Oeffnung der Contacte - sie mögen kur\% die Nullpunkte heissen - mittels Versetzung 
der Contactstopfen in andere Löcher der Rheotomscheibe und durch horizontale Verschiebung der Contactbänke an jede beliebige bestimmte Stelle des Cylinderumfangs verlegt werden können und, einmal fixirt, eine unveränderliche Lage behalten. Letzteres ist ganz streng begreiflicherweise nur bei Benutzung der festen Metallcontacte (Kupferfedern, Schliissel) der Fall. Bürsten- oder Pinselcontacte und in noch höherem Grade Quecksilbercontacte wirken weniger genau. Uebrigens ist es auch bei Benutzung der Kupferfedern wïnschenswerth, vor Anfang des Versuchs nach Einstellung der Federn und der Contactbänke den Cylinder mit der Rheotomscheibe einige Umdrehungen machen zu lassen, da die Kupferfedern anfangs wegen ihrer nicht absolut vollkommenen Elasticität noch ein wenig ibren Stand verändern können, wenigstens weun sie mit viel Reibung über die Contactbänke gleiten.

Die Bestimmung und Markirung der Lage der Nullpunkte auf dem Cylinderumfang geschieht nach demselben Princip wie bei v. Helmholtz' Myographion.

Bei einfacher Rotation des Cylinders durch Federmechanismus oder Uhrwerk und fester Einstellung der Contacte fallen - wie Fig. 1, 2, 3, 7, 9, 10 auf Taf. II belegen - die Nullpunkte bei beliebig oft wiederholter Umdrehung genau aufeinander, da auch der Cylinder auf seiner Axe unverrückbar festgestellt werden kann und die Axe keine merkliche Torsion erfährt. Beim Verschieben des Cylinders längs der Axe mit der Hand (Fig: 3, 6, 8) und - obschon etwas weniger vollkommen - auch bei der Spiralbewegung (Fig. 9) kommen die Nullpunkte genau vertikal über einander zu liegen, da durch geeignete Führungen gesorgt ist, dass der Cylinder bei der verticalen Verschiebung nicht eine Drehung um die Axe erfährt.

Durch dies Alles, in Verband mit der Constanz der Bewegung, lässt sich allen Versuchen und besonders längeren Versuchsreihen eine nicht genug $\% u$ schätzende Anschaulichkeit und Uebersichtlichkeit ertheilen, während zugleich die mathematische Regelmässigkeit der unter gleichartigen Bedingungen gezeichneten Curven und Curvenscharen mit einem Blick die ausserordentliche Präcision und Zuverlässigkeit der Leistungen des Apparats erkennen lässt. In der Dissertation von Boekelman sind schon eine Reihe von Belegen hierfür beschrieben und abgebildet. Doch geben die Abbildungen, weil nicht mechanisch reproducirt, die Originale nicht 
hinreichend genau wieder. Eine Anzahl anderer Belege enthält Taf. II, Autotypien einer Reihe von Kymogrammen, deren photographische Aufnahme ich der gitigen Vermittelung meines Collegen Professor E. Rosenberg in Utrecht verdanke.

\section{Erklärung der anf Tafel II}

abgebildeten Versuche zur Prüfung der Leistungen des Pantokymographion.

Alle Figuren sind auf $3 / 7$ der Originalgrösse photographisch verkleinert. Zur Vergleichung ist oben ein Centimetermaassstab mitphotographirt. Die den einzelnen Figuren beigeschriebenen Werthe für die Dauer von $1 \mathrm{~mm}$ Abscissenlänge oder für die Länge einzelner Stimmgabelschwingungen beziehen sich auf die Originale. Fig. 1-8 sind unter Benutzung der Federmechanik, Fig. 9-11 bei Bewegung mittelst Uhrwerk geschrieben. Mit 0 sind stets die Nullpunkte, d. i. die Momente der Schliessung bez. Oeffnung des electrischen Stroms durch den Apparat bezeichnet.

Fig. 1. Prüfung der Constanz der Nullpunkte unả der Bewegung des Cylinders. - Federmechanik. Treibstift in Loch $12+6$. Oeffnung eipes galvanischen Stroms, mittels Pfeils Signal 20 mal nacheinander an derselben Stelle derselben Abscisse registrirt. Stimmgabel (von50 in 1") nur zweimal aufgeschrieben. Geschwindigkeit der Sehreibfläche zur Zeit der Stromöffnung 1,75 Meter. Trotz 20 facher Superposition besteht die vom electrischen Signal gelieferte wellenförmige Zeichnung in ihrer ganzen Ausdehnung in einer einfachen, haarscbarfen Linie, deren Dicke, kaum grösser als bei nur einmaligem Registriren, im Original durchschnittlich etwa $0,1 \mathrm{~mm}$, an vielen Stellen noch weniger beträgt. Die Präcision des Apparats ist also jedenfalls so gross, dass Zeitdifferenzen von mehr als $1 / 20000$ Sekunde der Beobachtung hier sicher zugänglich erscheinen. Durch Anwendung noch feinerer Stahlspitzen zum Schreiben und bei dünmerer Berussung des Glanzpapiers kann die Empfindlichkeit noch auf etwa das Fünffache gesteigert werden. Doch sind solche Kymogramme, deren ich viele besitze, für heliographische Reproduction vorläufig zu zart.

Fig. 2. Aehnlicher Versuch, mit dem Unterschiede, dass nach je 5 Wiederholungen die Contactbank, welche den die Oeffnung besorgenden Stromschlüssel trägt, tangential etwas versehoben wird. Hierdurch sind $8 \mathrm{Cur}-$ ven in Juxtaposition oder "lateraler Imbrication" (Marey) gexeichnet, von denen jede durch Jeckung von fünf identischen Curven erhalten ist. Die Stimmgabel (50 in 1") ist nur einmal verzeichnet. Geschwindigkeit der Schreibfläche im Augenblick der Stromöffnung 1,35 Meter. - Die Deckung der 5 Curven ist in allen 8 Fällen in der ganzen Ausdehnung eine absolute; selbst bei mikroskopischer Prüfung der Originale ist keine Andeutung davon zu bemerken, dass mehr als einmal registrirt wurde. 
Fig. 3. Dasselbe mit verticaler Imbrication. Nach je 5 Versuchen wird der den Schlüssel öffnende Stopfen in der Rheotomscheibe zwei Löcher weiter versetzt. Nachdem dies 8 mal wiederholt ist (nur 5 dieser Wiederholungen sind abgebildet), wird der Cylinder mit der Hand um $1 / 2 \mathrm{~cm}$ auf der Axe des Cylinders herabgeschoben und eine neve Reihe von $8 \times 5$ Versuchen in derselben Weise wie zuvor angestellt. Jede der abgebildeten 40 Curven ist also durch 5 malige Superposition desselben Vorgangs erhalten. Stimmgabel (50 in $1^{\prime \prime}$ ) einmal aufgeschrieben. Geschwindigkeit der Schreibfläche auf der abgebildeten Strecke von $1,05 \mathrm{~m}$ bis $1,10 \mathrm{~m}$ wachsend. - Man bemerke ausser der vollkommenen Deckung der zusammengehörigen Curven auch die sehr genaue vertikale Superposition der Curven auf den übereinander stehenden Abscissen.

Fig. 4. Laterale Imbrication der Contractionen eines curarisirten ausgeschnittenen Froschgastrocnemius. Wiederholte Reizung durch einen Oeffungsinductionsschlag: 4 Kupferfedercontacte auf einem Umgang (nur 2 Rejzstellen abgebildet). Zwischen je 2 Umgängen eine Pause von etwa 10 Sec., worin die Feder auf's Neue gespannt und die Contactbank ein wenig verschoben wird. Geschwindigkeit der Schreibfläche auf der abgebildeten Strecke von $0,43 \mathrm{~m}$ (links) auf $0.39 \mathrm{~m}$ abnehmend.

Die Figur zeigt in sehr anschaulicher Weise die Abnahmè von Zuckungshöhe und Geschwindigkeit der Erschlaffung mit fortschreitender Ermüdung.

Fig. 5. Laterale Imbrication von Zuckungscurven durch Ver. setzen der Contactstopfen in der Rheotomscheibe. Messung der Fortpflanzungsgeschwindigkeit im motorischen Nerven. Zwölf Paare von Zuckungen eines blutdurchströmten Gastrocnemius auf derselben Abscisse, erhalten durch Reizung des Ischiadicus mit einem Oeffnungsinductionsschlag an je zwei verschiedenen, 5 bezüglich $55 \mathrm{~mm}$ vom Muskel entfernten Stellen, in Pausen von 15-20 Sec. - Die Geschwindigkeit der Schreibfläche wächst auf der abgebildeten Strecke von $0,4 \mathrm{~m}$ (links) auf $1,1 \mathrm{~m}$, um danach wieder abzunehmen.

Fig. 6. Vertikale Imbrication von Muskelzuckungen. Priifung der Constanz der Bewegung und des Azimuths der Nullpunkte bei Verschieben des Cylinders längs der Axe mit der freien Hand. - 16 Zuckungen eines blutdurchströmten curarisirten Froschwadenmuskels, erregt durch Oeffnungssebläge von gleicher submaximaler Stärke. Nach jeder Zuckung wird der Schreibhebel des Muskels um eine Spur höher geschraubt und bei offenem primären Kreis eine horizontale Abscisse gezeichnet, danach der Cylinder um 0,5 cm mit der Hand gesenkt. - Die 16 Momente der Reizung sind durch eine grade Linie $(0-0)$ verbunden, ebenso die Endpunkte des Stadiums latenter Erregung. Beide Linien laufen einander parallel und genau vertical. Auch die 16 Zuckungen sind in ihrem ganzen Verlauf nahezu vollkommen parallel. 
Fig. 7. Constanz der Nullpunkte und der Bewegung, demonstrirt durch zehnmalige doppelte Superposition von Contractionen eines Froschgastrocnemius. - Messung der Fortpflanzungsgeschwindigkeit im motorischen Nerven. - Derselbe Muskel, welcher Fig. 5 gezeichnet hat. Geringe Ueberlastung. Hebelvergrösserung $10 \mathrm{mal}$. Die Figur zeigt zwei Curven von völlig identischer Gestalt und Grösse, aber gegen einander um mehrere Millimeter verschoben, da die erste durch (10 mal wiederholte) Reizung des Ischiadicus in etwa $5 \mathrm{~mm}$ Entfernung vom Knie, die zweite durch Deckung von 10 Zuckungen in Folge Reizung des Nerven $50 \mathrm{~mm}$ weiter oben, dicht am Rückenmark, entstanden ist. Die Dicke der Curven ist nur im Stadium sinkender Energie sehr merklich grösser, als sie nach bloss einmaligem Registriren gewesen sein würde (vgl. Fig. b); aber erst an den unregelmässigen Nachschwingungen, welche der Muskelhebel nach dem Aufschlagen auf die Unterstiitzung ausführt, ist ersichtlich, dass es sich um mehr als je eine Zuckung handelt: im Original erkennt man gleichwohl auch bei Betrachtung mit starker Loupe mit Sicherheit nur 7 von den 20 in Wirklichkeit gezeichneten.

Die Ausmessung der Originale im Stadium der steigenden Energie ergibt im vorliegenden Falle beiläufig eine Fortpflanzungsgeschwindigkeit der motorischen Erregung im Nerven von $15,2 \mathrm{~m}$ mit einem wabrscheinlichen Febler von weniger als $\pm 0,4 \mathrm{~m}$.

Fig. 8. Verticale Imbrication von 40 Zuckungspa aren, erhalten durch abwechselnde Reizung des Ischiadicus in 5 und in $55 \mathrm{~mm}$ Entfernung vom Gastrocnemius, durch einen Oeffnungsinductionsstrom. Messung der Fortpflanzungsgeschwindigkeit der Firregung im motorischen Nerven.

Nach jedem Doppelversuch wird eine Abscisse ohne Reizung gezeichnet und dann der Cylinder um $0,5 \mathrm{~cm}$ mit der Hand gesenkt. Geschwindigkeit der Schreibfläche im Anfang der Zuckungen 0,72 m. - Man bemerke die nahezu vollkommene Identität in Form und Dimensionen der 80 Curven, die im Stadium steigender Energie constante seitliche Distanz der beiden zu jedem Versuchspaar gehörigen Zuckungen, welche einer Zeitdiferenz von durchschnittlich $0,00274^{\prime \prime}(=25,4 \mathrm{~m}$ Fortpflanzungsgeschwindigkeit) entspricht endlich die genau verticale Superposition der 40 Versuchspaare.

Fig. 9. Constanz der Bewegung und der Nullpunkte bei A nwend ung des Uhrwerks. Cylinder auf der Axe festgeschraubt. $8 \mathrm{~kg}$ über 3 Rollen, kleine Windfügel fast tangential gestellt, schnelles Wechselrad. Geschwindigkeit der Schreibfläche, bleibt nach 1/2 Umdrehnng constant $100 \mathrm{~mm}$ in 1“. Nach einmaliger Umdrehung führt der Cylinder noch 10 Rotationen aus, wobei ein Gastrocnemius je 6 mal durch einen Schliessungs - (o) und Oeffnungsinductionsschlag $\left(o_{1}\right)$ mittels Kupferfedercontact gereizt wird. Nur drei Reizstellen des Umganges sind abgebildet. Jede zeigt die durch 10 fache Superposition erneugto zusammengesetzte Zuckmgscurve als einfache 
Linie. Nur am Ende des Stadiums der sinkenden Energie ist in Folge der Ermüdung die Deckung nicht mehr vollkommen, an der zweiten Reizstelle weniger als an der ersten, an der dritten weniger als an der zweiten.

Fig. 10. Bewegung durch das Uhrwerk. Cylinder auf Axe festgeschraubt. Geschwindigkeit der Schreibfläche $75 \mathrm{~mm}$ in $1^{\prime \prime}$. Zehnmalige Reizung eines Gastrocnemius an der nämlichen Stelle (o) des Cylinderumfangs durch einen Oeffnungsinductionsschlag. Mässige Ueberlastung. Zwischen je zwei Reizungen wird die Muskelklemme etwas tiefer geschraubt, so dass der Muskel sich jedesmal um einen grösseren Betrag seiner Länge verkürzen muss, ehe er auf den Hebel wirken kann. Solche Contractionen, bei denen die Fasern sich erst gerade strecken müssen, ehe sie den Hebel in Bewegung setzen können, mögen hy o metris che heissen und der Grad $\mathrm{der}$ Hy p o metrie mag dem Betrag der ohne Wirkung auf den Hebel erfolgten Längenabnahme der Fasern proportional gesetzt werden. Das Gesetz, welches Fig. 10 mit einem Blick anschaulich macht, lässt sich dann so ausdrücken: mit wachsender Hypometrie nimmt die Kraft der Verkürzung während der Zuckung langsamer zu, erreicht aber früher ihr Maximum.

In ähnlich einfacher und übersichtlicher Weise lassen sich mittels unseres Apparats die gesetzlichen Beziehungen zwischen Eintritt, Verlauf und Grösse der Muskelcontraction einerseits und Art, Stärke, Zahl der Reize, Art und Grösse der Belastung (Isotonie, Isometrie, Auxotonie), Temperatur, chemischer Beschaffenheit des Muskels u. s. w. graphisch veranschaulichen. Einige Beispiele hierfür s. bei Boekelman Taf. VIII, Fig, 5 und 6 (Einfluss der Reizstärke auf die Dauer des Latenzstadiums), Fig. 8 (Einfluss der Ermüdung auf Grösse und Form der Verkürzung).

Fig. 11. Automatische Spiralbewegung des Cylinders vermittels des Uhrwerks. Kleinste Spule: Neigung der Spirale $1^{\circ}$; Rotationsgeschwindigkeit $100 \mathrm{~mm}$ in $1^{\prime \prime}$. 8 Kupferfedern in gleichen Abständen über die Rheotomscheibe vertheilt schliessen 8 mal während jedes Umgangs auf je etwa 0,8 Sec. Zeit einen galvanischen Strom. Schliessung und Oeffnung werden durch Pfeil's Signal registrirt, darunter Stimmgabelschwingungen von 10 in $1^{\prime \prime}$. Der Raumersparniss wegen ist nur ein Streifen aus dem Bogen abgebildet, der eine Gruppe von 22 übereinanderliegenden Markirungen des Signals zeigt. Die Nullpunkte liegen zwar nicht so vollkommen genau vertical übereinander, wie bei Versetzen des Cylinders längs der Axe mit Hand und Schraube, aber doch immerhin so genau, wie bei Versuchen, wo die Spiralbewegung überhaupt angebracht ist, nöthig scheint. Dieselbe Figur bei B oekelman Taf. VIII. Fig. 9 lithograpbisch copirt. 